\title{
Research in virtual home roaming system based on virtools
}

\author{
Hui $\mathrm{LI}^{1}$; \\ School of Computer and Informaiton Technology, \\ Northeast university of petroleum;NEPU; \\ Daqing,Heilognjiang, 163318,China; \\ e-mail: lihui_dqpi@163.com
}

\begin{abstract}
This system uses three dimensional scene roaming technology into home design, breaks the current rendering home design mode. It constructs $3 \mathrm{~d}$ indoor scene and indoor furniture models through $3 \mathrm{~d}$ modeling, and realizes the roaming interaction of $3 d$ home roaming scene by scene roaming technology, at the same time the system adopts collision detection technology to ensure the real roaming effect. The effect of roaming system can satisfy the client's need of knowing home pattern furthest, enhance the client's satisfaction degree of home pattern and reduce the burden and pressure of home designer's amending effect picture once and again.
\end{abstract}

Keywords-Roaming; 3D modeling; Virtools ; Collision detection

\section{INTRODUCTION}

Virtual reality technology is a new branch of subject in information science, it is widely used in military, entertainment, manufacture, medicine, geographic information system, etc, because of its peculiar advantage. Virtual reality technology will generate more real simulation environment by computer, the user immerses in a virtual environment, at the same time interacts with virtual environment directly through the man-machine interface [1]. Virtual scene cannot be matched with the traditional twodimensional rendering, $2 \mathrm{~d}$ and $3 \mathrm{~d}$ animation techniques in immersive, interactivity and real-time.

Currently home decorating designer mainly show rendering for customer to reveal its future household pattern and style, so a price - rendering time must be payed to achieve good performance. It is impossible to reach every aspect of a matter, it cannot be very good performance for the customer, sometimes it is difficult to satisfy our customers. In order to meet customer needs maximum, reduce the burden and pressure of the household designers, and improve the working efficiency of the household designers, make its more time in design and not waste time in rendering, we design and realize a virtual home roaming system based on virtools.

\section{VIRTUAL ROAMING SYSTEM OVERVIEW}

Virtual roaming system is a virtual environment with vision, hearing and touch in a particular range, it is a realtime space or imaginary space of the real-time simulation virtual space. Users roam naturally in the virtual space with the necessary equipment, observe object in the environment

\author{
Fang $\mathrm{LIU}^{2}$ \\ School of Computer and Informaiton Technology, \\ Northeast university of petroleum;NEPU; \\ Daqing,Heilognjiang, 163318,China;
}

from any angle, thus they feel as if they are there themselves, also can plan and operate the object [2].

Virtual scene roaming is a field of technology that is promising and more and more widely used. It is applied in architectural design, urban and rural planning, home decoration and so on, in virtual war practice field and combat command simulation training, in game design and entertainment industry, and in promoting the future new art form, etc, and it represents the industry's new technology and new level.

Roaming system realization includes two parts: visual database generation and roaming platform design. The process of roaming system realization is shown in figure 1. Among them, the visual database generation includes three dimensional model structure and the media (including images, voice) generation. Roaming driver design included 3d graphic display and input command control.

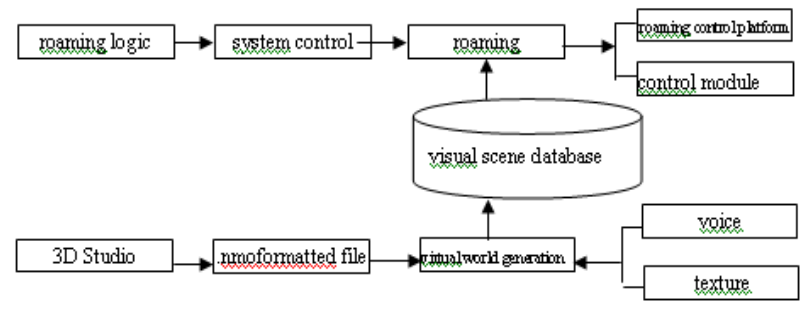

Figure 1 rasming system generation process

III. DESIGN AND IMPLEMENTATION OF VIRTUAL HOME ROAMING SYSTEM

The virtual home roaming system discussed in this paper is realized by 3DS MAX to complete household scene and construct the model, it uses multifunctional 3d development tools--Virtools to realize the whole system roaming engine.

\section{A. The system overall design goal}

The virtual home roaming system based on Virtools embodies the characteristics of free roaming, so users can real-time interaction with the system, control the line of sight from the before and after ,steering, pitching and so on through the keyboard to any places they want to reach. They also can fully observe in each angle, integrally understand future household environment and household style. This system can make designer and client communicate better. Home roaming system is a complicated application project, so we focus on home roaming system $3 \mathrm{~d}$ models building 
and household roaming system design and implementation by Virtools.

\section{B. System model building}

Modeling technology is a principle and method to convert objects and their attributes in real world into internal computer digital expression [3]. Three dimensional modeling technology is one of key techniques in roaming system. The world that we are roaming whether "looks real, moves real, feels real" or not most relies on modeling technology. Modeling has a large proportion in the whole system design process, the model design concept and the type of design style are closely related to the success of a work. The preparing work before constructing model includes field data collection (i.e. measuring scale), painting CAD diagram, model building and model beautification.

\section{- $\quad$ Field data collection (i.e. measuring scale)}

In order to indoor design accurately and intuitively as possible, we need to inspect the scene, measure actual size of the roughcast house by meter scale, and then make a certain proportion shrinkage change according the object size in real world, we can form a fixed measuring scale, the purpose is to zoom each part of the model in the same proportioning, so that the model looks real. We use grid in 3DS MAX to model in order to increase the accuracy of model.

- Painting CAD diagram

Painting CAD diagram combined with the user's housing type.

\section{- Model building}

When the above work is completed, we begin to create model in 3DS MAX, the modeling order should be from whole to local, from rough to detail, slowly beautification model and finally achieve the ideal effect, at last combine all the model formation into a synthesis scene.

When constructing the system, we derive the painted CAD model in DWG format, import this model into 3DS MAX. Firstly build the wall, and then divide the space of the wall into different functional area, like sitting room, bedroom, kitchen, toilet, etc. Finally furniture model is imported to these areas, using the merging method.

- Material establish and mapping

When all the modeling is completed, we observe that the model fidelity is not very high, it is necessary to increase model texture, color and texture by mapping. Attention must be paid to observe the character of objects in real world, and create a sense of reality material according to the special properties. In 3DS MAX, material and texture is established and edited by material editor-Material Editor, and through the final render to display them, so the object surface shows different quality of a material, color and texture. Material in the $3 \mathrm{~d}$ model creation process is crucial part, we increase the details of the model through it, which reflects the model detail, and has a direct impact on how to establish object model.

- Modeling problems that should be paid attention to

1. Modeling process should pay attention to the use of plane

We need to reduce the use of plane meanwhile keep the objects alike, so the object edge and section number should be smaller. If the object surface number is too much and cannot be reduced, then MultiRes in modifier need to use to reduce its plane in the condition that its effect is not affected.

2. Modeling should make use of object selection and capture

The use of object selection and capture in 3DS MAX operation plays a decisive role (especially in making fine model).

3. Modeling should pay attention to coordinate proportion of each object

The proportion coordinating object modeling can increase the $3 \mathrm{~d}$ scene sense of reality.

\section{Model optimization}

Usually model optimization in 3DS MAX environment is to remove redundancy polygons. If in model construction we use the least amount of plane to get the same effect, it is the most successful model. Usually, initial model may have some unnecessary polygon, in this case, the model data volume increases, the real-time display performance is reduced, and user's visual phenomenon is that the movement process of model in $3 \mathrm{~d}$ virtual scene will frequently appear "blink" phenomenon. Therefore polygon which will not appear in the vision needs to be removed. Such operation should be done after $3 \mathrm{~d}$ model preliminary completed, because in modeling process there will be some polygons as the other parts of auxiliary plane to construct a three dimensional model. Finally, after the model is completed, we check that whether there are redundant planes, if there still are some, we need to manually delete them.

\section{THE SCENE REALIZE OF HOME ROAMING SYSTEM}

\section{A. Home $3 D$ scene integration by Virtools}

a) The output model format from 3DS MAX is .nmo Open 3DS MAX files, let the scene contains only a model object, click on the 3DS MAX File/Export menu commands above. User define the storage path and file name, but it is strongly suggested using English when naming, or there will be unexpected error occurs when file import to Virtools. Please select Virtools Export (*. NMO, *. VMO) as the file saving type, then confirm of preservation.

\section{b ) Import Virtools special model}

The next step is to open Virtools and import document. Start Virtools, enter the working interface, select the top menu commands -- Resources / Import File, identify and open the saved NMO files, you can see the model done by 3DS MAX has successful import to Virtools, and then put and arrangement the import model. 


\section{B. Home 3d scene beautification modification by Virtools}

This step is primarily for light setting in $3 \mathrm{~d}$ scene and adjustment of 3d model material, it can let whole 3d scene and its model achieve optimal effect display.

\section{Home effect show}

Here we import the model to Virtools, and go on with the corresponding beautification after modification. The part of system rendering as shown in figure 2,3 .

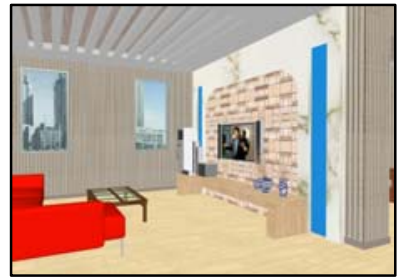

Figure 2 design styleof the sitting room

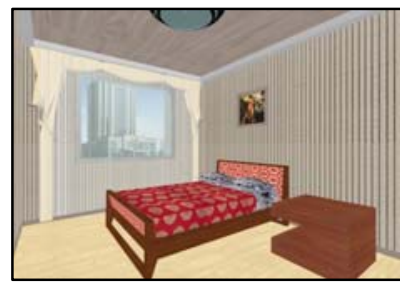

Figure 3 design styleof the bedroom

\section{REALIZATION OF THREE DIMENSIONAL ROAMING ENGINE IN HOME ROAMING SYSTEM}

\section{A. Function of the roaming engine}

Virtual scene roaming system need to provide a variety of roaming control function for interaction between user and the virtual scene. Free roaming control features realized in this paper include: the sight forward, backward, around translation, rotation, free roaming in the $3 \mathrm{~d}$ scene roaming, etc. The functions of forward, backward, around translation, rotation, free roaming are controlled by sight that point form viewpoint to reference point. In the scene a simulation user view point (namely view) is built, when the observation point position and the line of sight direction changes, the observed scene part will also changes. After the system gets the changed view, it changes parameter, repaints scene, so simulates the user's observation in different position, different point of view scene [4]. The realization of the home roaming is primarily through $\mathrm{BB}$ interaction module in Virtools, the realization process is relatively complex, so we need to have a certain understanding in modules and each modules attribute before system use.

\section{B. Free roaming}

Free roaming in three dimensional scene, that is user observe from different angle and azimuth through input control. The main input method in this system is keyboard. We can complete users' different action through the function keys on keyboard, for example, forward, backward, around rotating, left, right, etc. When system gets keyboard commands, it changes the corresponding parameters, and repaints scene.

Its principle is: preassign fixed function keys and define the corresponding operation detail, when the user press the corresponding function key, system obtain action information of the function key, and pass it on to the scene rendering module. Scene rendering module get action information, call the corresponding function, modify the corresponding position parameters, repainting scene with a new parameter requirements through the geometric transformation. Users do all kinds of operatation continuously, the scene is redrawed and displayed in different angle and pose.

When the observers move forward (that is close to the screen direction), object translate according to the $\mathrm{z}$ axis positive direction; the observer move backward (away from the screen direction), object translate according to the $\mathrm{z}$ axis negative direction. The observer turn right, the object translate according to the $\mathrm{x}$ axis negative direction; The observer turn left, the object translate according to the $\mathrm{x}$ axis positive direction. When observers observe around in horizontal surfaces, object rotate around the $y$ axis of the clockwise and anti-clockwise.

As shown in figure 4, Switch On Key BB defines each function keys and is responsible for the call each Key corresponding action. View mobile and rotating speed is controlled by Per Second BB, it calculates moving vector or rotation angle of each frame viewpoint according to the parameters setting, and inputs and outputs to corresponding parameters of Translate $\mathrm{BB}$ or Rotate $\mathrm{BB}$, so it controls viewpoint mobile and rotating speed. Let us suppose the input to Per Second $\mathrm{BB}$ is $\mathrm{X}$, output is $\mathrm{Y}$, then their relationship is $\mathrm{Y}=\mathrm{X} *$, it is the time to render a frame of image.

\section{Key technology in three dimensional roaming}

1. Realization of camera move forward and around and up and down

According to the requirement of roaming, camera must have the function of movement and rotating under the control of people.

Step one: the camera's forward and backward

(1) Select the camera;

(2) Set the camera as a role;

(3) The up and down, left and right key in keyboard is used to control the role before and after, left and right, Page up key makes camera move up, Page down makes camera move down.

Step two: Camera rotation around

(1) Select the camera;

(2) Set the camera as a role;

(3) The Z, C key in keyboard is used to control the rotation of the role.

The right demo of the integrated camera operation module is shown in figure 4.

2. Collision detection of camera and wall

In the 3d applications, people most do not want to see unreality when roaming through object. Often part body of the role appears in the walls, sometimes the role does not touch the wall but can't continue to move. In order to simulate the real world in the virtual environment, it is necessary to restrict the user's mobile and the moving object in virtual world. Collision detection is a kind of commonly 
used methods. By means of collision detection, it can avoid the user plunged underground or the building celerity when roaming walk, it also can avoid moving object fusion, so as to realize a similar sense of reality in the real world of [5]. All these prove importance of correct collision detection technology in 3d application.

After this system has constructed above, there still exist the problem of camera through wall. It directly leds to the problem in manual operation that user will roam into another room when roam in a certain room. In order to solve the problem of camera through wall, this system uses the collision detection module that Virtools itself offered to ensure system roaming reality sense.

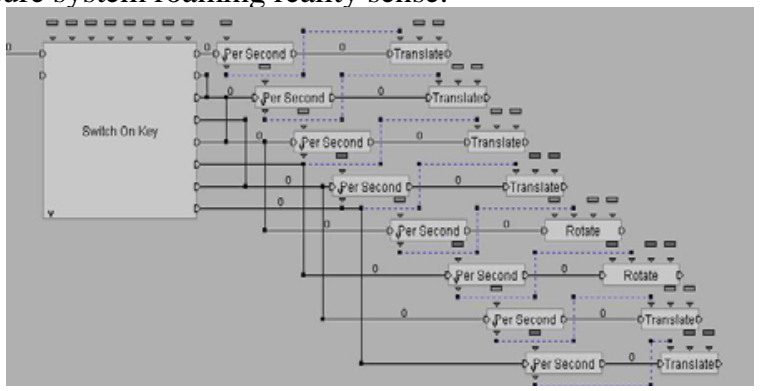

Fiqure 4 integrated camera

\section{CONCLUSION}

Using immersive and the sense of reality of virtual reality technology, we break the way of traditional home design for the customer, it can design home roaming system according to the customer's own needs. This manner is more interactive, more communicative, more modern, it makes customer has a feeling of be personally on the scene, as if place oneself in the future new home. Customers can walk free in the roaming space, observe every corner of the home, it is advantageous to communicate between the designer and customers, maximally improve customer satisfaction.

\section{ACKNOWLEDGMENT}

One reviewer made several helpful suggestions which I hope I have implemented satisfactorily to clarify this paper.

\section{REFERENCES}

[1] Zhou, Muxiong,Tan, Guoxin. Design and implementation of a virtual indoor roaming system based on Web3D. International Conference on Fluid Power and Mechatronics.2011:775-778,

[2] Zhang, Li,Zheng, Guang. A roaming system of the residential building's virtual internal scene based on VRML. 2010 2nd International Workshop on Intelligent Systems and Applications. 2010:571-475.

[3] Han, Hongling,Yang, Fenglei. Large-scale terrain scene modeling and roaming simulation based on VRML.2nd Conference on Environmental Science and Information Application Technology, ESIAT: 2010 .

[4] 4.Wu, Zhanwei,Wang, Heng,Zhang, Hua. Virtual scene modeling technology based on OpenGL and 3dsMAX.4th International Symposium on Computational Intelligence and Design, ISCID.2011: 170-173.

[5] Li, Ziwei,Yang, Gang,Cao, Weiqun. Design and implementation of virtual flower exhibition. Journal of Information and Computational Science, 2010:981-988. 